\title{
Desempenho Silvicultural de Tectona grandis L. f. em Diferentes Espaçamentos em Cáceres, MT
}

\author{
Raiane Scandiane da Silva ${ }^{1}$, Diogo Guido Streck Vendruscolo ${ }^{2}$, \\ José Renato Maurício da Rocha ${ }^{3}$, Arthur Guilherme Schirmbeck Chaves ${ }^{3}$, \\ Hudson Santos Souza ${ }^{2}$, Alessandro Soares da Motta ${ }^{3}$
}

${ }^{1}$ Departamento de Agronomia, Universidade do Estado de Mato Grosso - UNEMAT, Cáceres/MT, Brasil ${ }^{2}$ Departamento de Engenharia Florestal, Universidade Federal de Mato Grosso - UFMT, Cuiabá/MT, Brasil ${ }^{3}$ Departamento de Engenharia Florestal, Instituto Federal de Educação Ciência e Tecnologia de Mato Grosso - IFMT, Campus Cáceres, Cáceres/MT, Brasil

\begin{abstract}
RESUMO
A teca atende aos principais critérios de seleção das espécies florestais para a produção de madeira de rápido crescimento. A escolha do espaçamento adequado fornece maior volume por área e fustes com qualidade desejável. O objetivo deste estudo foi avaliar o efeito do espaçamento sobre diversas variáveis dendrométricas em povoamentos de teca em Cáceres, MT. Foram feitas avaliações nas idades de 11 e 16 anos referentes a: diâmetro a 1,3 m de altura; altura total; área basal; volume total por árvore; volume por hectare; incremento médio anual; e sobrevivência. Observou-se que quanto menor a densidade de árvores, maiores são os valores de DAP e volume individual e que quanto maior a densidade, maiores foram os valores de área basal e volume total por hectare. A ausência de desbastes afetou negativamente as medias dendrométricas e a produção dos povoamentos nos diferentes espaçamentos.
\end{abstract}

Palavras-chave: teca, densidade, produção.

\section{Silvicultural Performance of Tectona grandis L.f. at Different Spacings in Caceres, Mato Grosso State, Brazil}

\begin{abstract}
Teak meets the main criteria for selection of tree species for timber production of rapid growth. Thus choosing the proper spacing will provide the largest volume per area and stems with desirable quality. The objective of this study was to evaluate the effect of spacing in several dendrometric variables in stands of teak in Caceres, Mato Grosso state. The following variables were assessed at the ages of 11 and 16 years: diameter at breast height (DBH), total height, basal area, total volume per tree, volume per hectare, annual average growth, and survival. It was observed that the lower the density of trees, the greater the DAP and the individual volume; and that the greater the density, the greater the basal area and the total volume per hectare. Lack of thinning negatively affected the dendrometric averages and the production of stands at different spacings.
\end{abstract}

Keywords: teak, density, production. 


\section{INTRODUÇÃO}

A crescente demanda madeireira associada à exploração intensiva comprometeu os estoques das florestas nativas, evidenciando a necessidade de estudos para aumentar a produção de madeira a partir de florestas plantadas. No ano de 2012, o Brasil tinha 7.185.943 ha de plantios florestais de diversas espécies, em que cerca de 5.102 .030 ha $(71,0 \%)$ eram de eucalipto, 1.562.782 ha (21,7\%), de pinus e 521.131 ha $(7,3 \%)$, de outras espécies. Os plantios de teca (Tectona grandis) totalizaram apenas 67.329 ha $(0,9 \%)$ e localizavam-se principalmente nos estados de Mato Grosso, Pará e Roraima (ABRAF, 2013).

Dentre as espécies com potencial para reflorestamento e produção de madeira para serraria está a teca, cujos primeiros plantios no Brasil ocorreram no início do século XX, na região de Piracicaba, SP. No Mato Grosso, também considerado o estado propulsor dos plantios, o início do cultivo se deu nos anos 1970, sendo que a espécie apresentou bons resultados quanto ao seu desenvolvimento (Passos et al., 2006). Isto se deve principalmente às condições ambientais adequadas, $O$ que proporciona taxas de crescimento superiores às dos plantios da maioria dos países produtores de madeira dessa espécie (Passos et al., 2006; Pelissari et al., 2013).

A teca atende aos principais critérios de seleção das espécies florestais para a produção de madeira de rápido crescimento, fácil propagação e estabelecimento, além do alto valor econômico e resistência a pragas e doenças (Figueiredo, 2001; Camino \& Morales, 2013). A importância e o valor comercial da teca se devem às propriedades físico-mecânicas desejáveis da madeira que são: durabilidade, estabilidade, facilidade de pré-tratamento, resistência natural ao ataque de fungos, insetos, pragas e brocas. Além dessas, desenho, cor e densidade são aspectos qualitativos importantes que tornam a espécie uma das mais valorizada no mundo (Schuhli \& Paludzyszyn, 2010). No entanto, verifica-se que os povoamentos de Tectona grandis L. f. apresentam produtividade extremamente variável em decorrência da diversidade de condições físicas e nutricionais do solo, combinadas com os diferentes graus de tratos culturais (Shimizu et al., 2007).

Um dos requisitos que requer cuidado no momento da implantação de um povoamento florestal é a escolha do espaçamento, que tem sido discutido de vários estudos quando se trata da cultura da teca (Macedo et al., 2005; Passos et al., 2006; Souza, 2010).

O espaçamento ótimo é aquele capaz de produzir o maior volume de produto em tamanho, forma e qualidade desejáveis, sendo função da espécie, do sítio e do potencial genético do material que for utilizado (Scolforo, 1998). Além de ter influência marcante na produção, o espaçamento afeta significativamente os custos de implantação, manutenção e exploração da floresta, podendo afetar a qualidade final da madeira produzida (Scolforo, 1998).

Desse modo, o objetivo deste estudo foi avaliar o efeito do espaçamento sobre diversas variáveis dendrométricas em povoamentos de Tectona grandis em Cáceres, MT.

\section{MATERIAL E MÉTODOS}

\subsection{Caracterização da área de estudo}

O estudo foi conduzido no Instituto Federal de Educação, Ciência e Tecnologia de Mato Grosso, campus Cáceres $\left(16^{\circ} 11^{\prime} \mathrm{S}, 57^{\circ} 40^{\prime} \mathrm{W}\right.$ e altitude média de $130 \mathrm{~m}$ ) (Figura 1).

A área pertence à sub-bacia do alto do rio Paraguai, bacia do rio da Prata, Planície do Pantanal. A vegetação predominante no alto do rio Paraguai é representada por $30 \%$ de savana, $20 \%$ de florestas e $50 \%$ de vegetação típica de Pantanal, sendo a área originalmente coberta por savana arborizada (cerrado) (Passos et al., 2006). $\mathrm{O}$ relevo é plano e o solo predominante na região é do tipo Argissolo Vermelho-Amarelo distrófico, entretanto, na área em estudo, o solo é um Latossolo Vermelho-Amarelo distrófico (Passos et al., 2006).

A região apresenta clima tropical (Aw) segundo a classificação de Köppen, com inverno seco, verão chuvoso e temperatura média anual de $26^{\circ} \mathrm{C}$, podendo ocorrer temperaturas de até $41{ }^{\circ} \mathrm{C}$. A pluviosidade anual é de aproximadamente $1.335 \mathrm{~mm}$, concentrada principalmente nos meses de dezembro a março (Neves et al., 2011).

A área do experimento era anteriormente coberta por pastagem. O terreno foi preparado com duas passagens de grade pesada, enleiramento das touceiras de capim fora da área experimental e com uma passagem de grade leve para o nivelamento do terreno. 


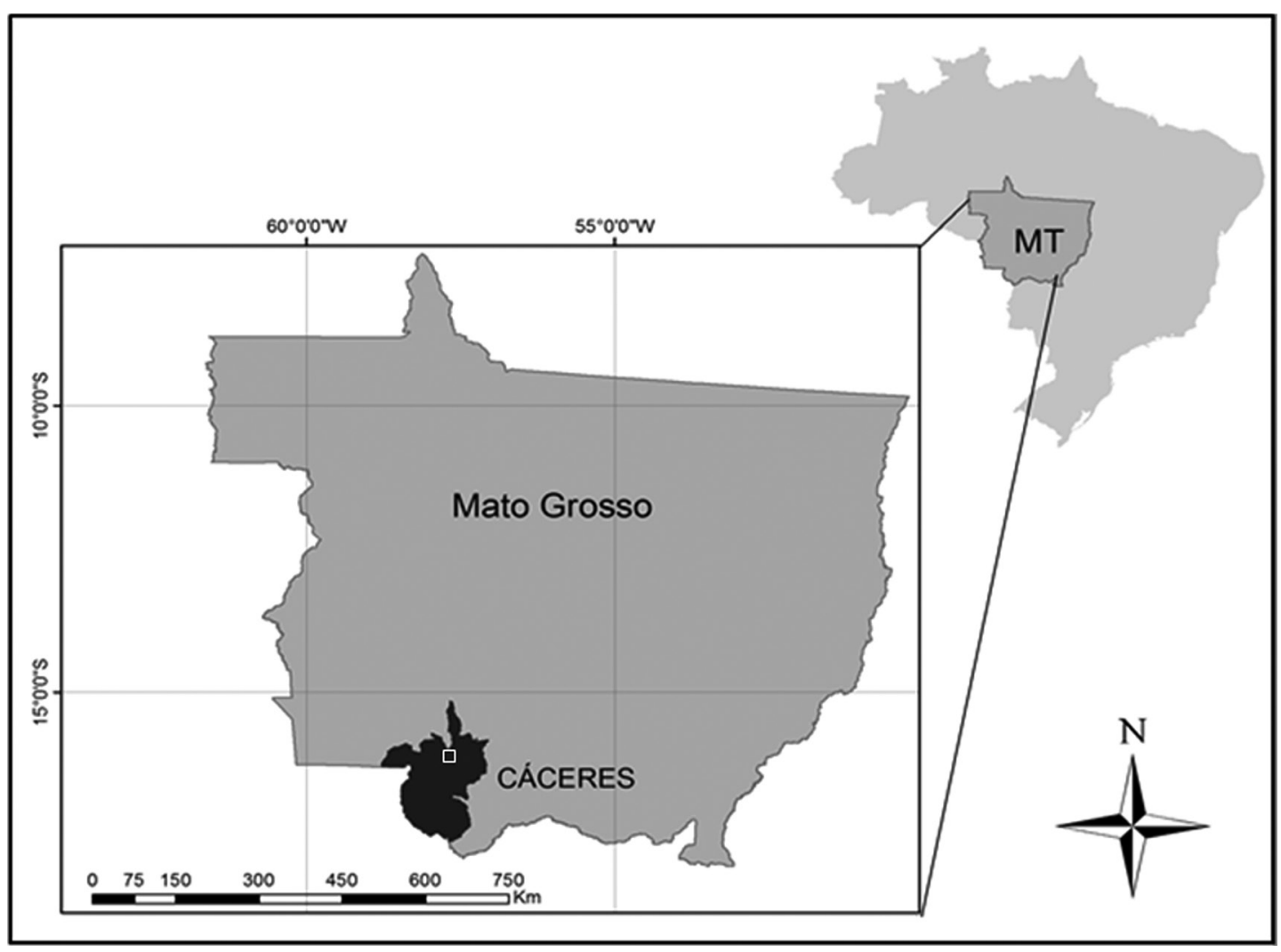

Figura 1. Localização dos povoamentos de Tectona grandis estudados.

Figure 1. Location of Tectona grandis plantations studied.

A teca foi plantada manualmente com mudas do tipo toco (stump) em dezembro de 1998, em covas de $40 \mathrm{~cm} \times 40 \mathrm{~cm}$, fertilizadas com $190 \mathrm{~g}$ de superfosfato simples e $10 \mathrm{~g}$ de Frited Trace Elements (FTE) BR-15. No primeiro ano foram realizadas manualmente três capinas e duas adubações de cobertura, uma aos 60 dias e outra no nono mês, cada uma com $95 \mathrm{~g} /$ cova de NPK 20-05-20, mais $5 \mathrm{~g} /$ cova de FTE BR-15.

As podas e desbrotas das árvores foram feitas aos 9, 14 e 22 meses de idade com tesoura de poda, serrote ou motosserra Still 025 , de acordo com a dimensão dos galhos. Foram feitas três capinas, aos 30 e 90 dias e aos 14 meses, e duas roçadas mecanizadas, aos 17 e 22 meses (Passos et al., 2006). Não foram realizados desbastes na área até o momento desta avaliação.

\subsection{Delineamento estatístico experimental}

O delineamento experimental utilizado foi de blocos ao acaso (DBC), com quatro tratamentos e três repetições. Os tratamentos foram os seguintes: espaçamentos
3 × 2 m (1.667 árv.ha $\left.{ }^{-1}\right), 4$ × 2 m (1.250 árv.ha $\left.{ }^{-1}\right), 5$ × 2 m (1.000 árv.ha-1 ${ }^{-1}$ e 6 × 2 m (834 árv.ha $\left.{ }^{-1}\right)$. As parcelas são formadas por cinco linhas de plantio com 32 árvores cada. O tamanho das parcelas varia de acordo com o tratamento, sendo: $960 \mathrm{~m}^{2}, 1.280 \mathrm{~m}^{2}, 1.600 \mathrm{~m}^{2} \mathrm{e}$ $1.920 \mathrm{~m}^{2}$ para os espaçamentos $3 \times 2 \mathrm{~m}, 4 \times 2 \mathrm{~m}$, $5 \times 2$ m e $6 \times 2$ m, respectivamente. Foram amostradas três fileiras centrais, sendo as demais utilizadas como bordadura individual para cada parcela.

\subsection{Coleta e processamento de dados}

A coleta de dados foi feita aos 11 e 16 anos de idade dos povoamentos. Aos 11 anos foram obtidos dados de diâmetro a 1,3 m de altura (DAP) e altura total (H) por meio de censo florestal realizado em todas as parcelas, já aos 16 anos foi realizado o censo florestal somente para a variável DAP, que posteriormente foi dividida em classes diamétricas com amplitude de $5 \mathrm{~cm}$. Foram coletadas as alturas de $20 \%$ das árvores de cada classe em cada espaçamento para o ajuste do 
modelo de relação hipsométrica proposto por Curtis (1967) (Equação 1), o que permitiu a predição das demais alturas que não foram mensuradas.

$\ln (H)=\beta_{0}+\beta_{1} \frac{1}{D A P}$

em que: $\mathrm{ln}=$ logaritmo neperiano; $\mathrm{H}=$ altura total; $\beta_{0}$ e $\beta_{1}=$ coeficientes do modelo; DAP = diâmetro a $1,3 \mathrm{~m}$ de altura.

Os ajustes foram feitos por tratamento, visto que o espaçamento pode afetar a relação entre a altura e diâmetro das árvores (Curtis, 1967). As equações foram avaliadas quanto ao coeficiente de determinação $\left(\mathrm{R}^{2}\right)$, erro padrão da estimava $\left(\mathrm{S}_{\mathrm{yx}} \%\right)$, à probabilidade do valor de $\mathrm{F}$ da análise de variância, significância dos coeficientes de regressão e análise gráfica dos resíduos em função do DAP. A obtenção dos volumes das árvores foi feita empregando-se as Equações 2, 3, 4 e 5 ajustadas por Vendruscolo et al. (2014) para os mesmos tratamentos na área de estudo.

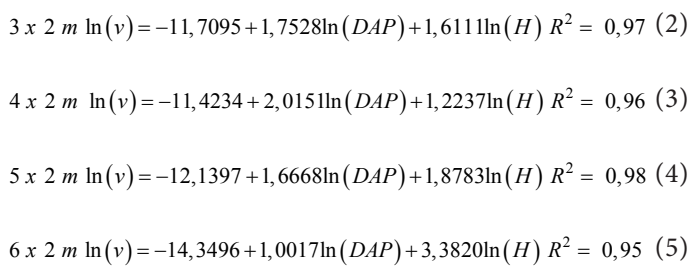

em que: $\ln =$ logaritmo neperiano; $\mathrm{v}=$ volume; $\mathrm{DAP}=$ diâmetro a $1,3 \mathrm{~m}$ de altura; $\mathrm{H}=$ altura total; $\mathrm{R}^{2}=$ coeficiente de determinação.

As variáveis analisadas neste estudo foram: o diâmetro a 1,3 m de altura (DAP); altura total $(\mathrm{H})$; área basal (G) em $\mathrm{m}^{2}$; volume total por árvore (v) $\mathrm{em} \mathrm{m}^{3}$; volume total de madeira por hectare $(\mathrm{V}) \mathrm{em} \mathrm{m}^{3}$; o incremento médio anual (IMA) em $\mathrm{m}^{3}$.ha ${ }^{-1}$; e porcentagem de sobrevivência (S).
Os dados foram submetidos ao teste de normalidade de Shapiro-Wilk, em seguida procedeu-se à análise de variância para tratamentos e blocos e posteriormente as médias foram comparadas pelo teste de Tukey a 5\% de probabilidade. O programa utilizado para as análises foi o ASSISTAT (Silva \& Azevedo, 2002).

\section{RESULTADOS E DISCUSSÃO}

\subsection{Predição da altura}

Após o ajuste das equações hipsométricas utilizadas para a predição da altura das árvores que não foram mensuradas na idade de 16 anos foi verificado que o modelo empregado apresentou desempenho satisfatório em todos os tratamentos. Os ajustes resultaram em coeficientes e valores de F significativos a 95\% de probabilidade, os valores de coeficiente de determinação $\left(R^{2}\right)$ oscilaram entre 0,56 a 0,83 e o erro padrão da estimativa $\left(\mathrm{S}_{\mathrm{yx}}\right)$ apresentou-se abaixo de $12 \%$ para todos os espaçamentos (Tabela 1).

Nos espaçamentos mais amplos ( 5 x 2 m e 6 × $2 \mathrm{~m})$ foi evidenciada uma diminuição da precisão nas estimativas, fato que, segundo Bartoszeck et al. (2004), é esperado à medida em que diminui a densidade de povoamentos florestais. Vários estudos demostram a eficiência do modelo de Curtis (1967) para relações hipsométricas com espécies florestais (Ribeiro et al., 2010; Oliveira et al., 2011; Vendruscolo et al., 2015). Avaliando-se a dispersão residual foi verificado que os erros se distribuíram de maneira satisfatória sem superestimar ou subestimar as alturas (Figura 2).

\subsection{Avaliação dos tratamentos aos 11 anos}

Os dados avaliados apresentaram distribuição normal de acordo com o teste de Shapiro-Wilk ao nível de $5 \%$ de probabilidade. Não foi verificada

Tabela 1. Coeficientes e estatísticas de ajuste e precisão para as equações hipsométricas ajustadas para cada tratamento.

Table 1. Coefficients and precision statistics for the hypsometric equations adjusted for each treatment.

\begin{tabular}{ccccrc|} 
Tratamentos & $\boldsymbol{\beta}_{0}$ & $\boldsymbol{\beta}_{1}$ & $\mathbf{R}^{2}$ & $\mathbf{S}_{\mathbf{y x}} \%$ & $\mathbf{F}_{\text {cal. }}$ \\
\hline $3 \times 2 \mathrm{~m}$ & $3,13295^{*}$ & $-7,41769^{*}$ & 0,82 & 6,64 & $326,08^{*}$ \\
$4 \times 2 \mathrm{~m}$ & $3,33537^{*}$ & $-10,5789^{*}$ & 0,83 & 7,94 & $438,86^{*}$ \\
\hline $5 \times 2 \mathrm{~m}$ & $3,11488^{*}$ & $-6,89387^{*}$ & 0,56 & 11,25 & $88,18^{*}$ \\
\hline $6 \times 2 \mathrm{~m}$ & $3,12217^{*}$ & $-7,28861^{*}$ & 0,65 & 7,60 & $134,32^{\star}$ \\
\hline
\end{tabular}

$\beta_{0} e \beta_{1}=$ coeficientes de regressão; $\mathrm{R}^{2}=$ coeficiente de determinação; $\mathrm{S}_{\mathrm{yx}} \%=$ erro padrão da estimativa; $\mathrm{F}_{\text {cal. }}=\mathrm{F}$ calculado; ${ }^{*}=$ significância a $5 \%$ de probabilidade $(\mathrm{p}<0,05)$. 
diferença significativa entre os valores médios das variáveis $\mathrm{DAP}, \mathrm{H}, \mathrm{v}, \mathrm{V}$, IMA e $\mathrm{S}$ (Tabela 2). Já a variável $G$ apresentou maiores médias nos espaçamentos mais adensados, sendo que o espaçamento $3 \times 2 \mathrm{~m}$ diferiu estatisticamente de $5 \times 2 \mathrm{~m}$ e de $6 \times 2 \mathrm{~m}$ e os demais proporcionaram efeitos iguais entre si. No entanto, cabe ressaltar que as maiores médias de DAP e v foram encontradas nos espaçamentos mais amplos. Rondon (2006) e Vendruscolo et al. (2015) relatam o mesmo efeito no crescimento em DAP em plantio de $T$. grandis. Os autores verificaram a ocorrência de maiores valores médios obtidos para tal variável nos tratamentos mais amplos. Já a variável $\mathrm{H}$ não foi afetada pelo espaçamento, como também verificado por Rondon (2006) para T. grandis.

Em relação ao volume médio individual por árvore (v), observou-se a elevação dos valores com o aumento do espaçamento, enquanto para área basal (G) e volume total por hectare (V) a relação foi inversa. A maior ocupação de $\mathrm{G}$ e o maior estoque volumétrico apresentaram-se nos espaçamentos mais adensados. Esse comportamento é esperado, segundo Scolforo (1998). Ou seja, em espaçamentos reduzidos, encontra-se maior área basal e volume de madeira por área do que naqueles espaçamentos mais amplos, em decorrência do maior número de árvores por hectare.

A sobrevivência das árvores aos 11 anos foi superior a $90 \%$ e não diferiu estatisticamente entre os tratamentos. No entanto, percebe-se que nos espaçamentos mais adensados foi menor o percentual de sobrevivência em campo, o que pode prejudicar a produção total. Krishnapillay (2000) cita que a competição por espaço e recursos é diretamente proporcional à densidade do povoamento; o autor afirma ainda que a teca sob
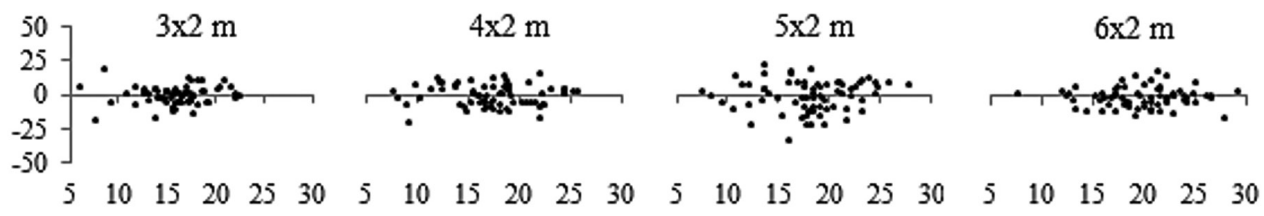

Figura 2. Distribuição dos resíduos em percentagem (eixo y) em função do diâmetro a 1,3 m de altura (eixo x) para Tectona grandis em diferentes espaçamentos em Cáceres, MT.

Figure 2. Residual Distribution percentage (y-axis) in function of diameter at $1.3 \mathrm{~m}$ height (x axis) for Tectona grandis at different spacings in Cáceres-MT.

Tabela 2. Valores médios de diâmetro a 1,3 m de altura (DAP), altura (H), área basal (G), volume individual (v), volume total por hectare (V), incremento médio anual (IMA) e sobrevivência (S) de Tectona grandis aos 11 anos em Cáceres, MT.

Table 2. Mean values of diameter at $1.3 \mathrm{~m}$ height $(\mathrm{DBH})$, height $(\mathrm{H})$, basal area $(\mathrm{G})$, individual volume (v), total volume per hectare (V), mean annual increment (MAI) and survival (S) Tectona grandis at 11 years old in Cáceres-MT.

\begin{tabular}{|c|c|c|c|c|c|c|c|}
\hline \multirow[b]{2}{*}{ Tratamentos } & \multicolumn{7}{|c|}{ Variáveis } \\
\hline & $\begin{array}{l}\text { DAP } \\
(\mathrm{cm})\end{array}$ & $\begin{array}{c}\mathrm{H} \\
(\mathrm{m})\end{array}$ & $\begin{array}{c}\mathrm{G} \\
\left(\mathrm{m}^{2} \cdot \mathrm{ha}^{-1}\right)\end{array}$ & $\begin{array}{c}\mathbf{v} \\
\left(\mathbf{m}^{3}\right)\end{array}$ & $\begin{array}{c}\mathrm{V} \\
\left(\mathrm{m}^{3} \cdot \mathbf{h} \mathbf{a}^{-1}\right)\end{array}$ & $\begin{array}{c}\text { IMA } \\
\left(\mathbf{m}^{3} \cdot \mathbf{h a}^{-1}\right)\end{array}$ & $\begin{array}{c}S \\
(\%)\end{array}$ \\
\hline $3 \times 2 \mathrm{~m}$ & $14,8 \mathrm{a}$ & $12,7 \mathrm{a}$ & $26,3 \mathrm{a}$ & $0,0604 \mathrm{a}$ & $93,0 \mathrm{a}$ & $8,46 \mathrm{a}$ & $92,2 \mathrm{a}$ \\
\hline $4 \times 2 m$ & $16,1 \mathrm{a}$ & $12,3 \mathrm{a}$ & $23,4 \mathrm{ab}$ & $0,0708 \mathrm{a}$ & $81,1 \mathrm{a}$ & $7,37 \mathrm{a}$ & $93,1 \mathrm{a}$ \\
\hline $5 \times 2 \mathrm{~m}$ & $16,4 \mathrm{a}$ & $13,5 \mathrm{a}$ & $20,2 \mathrm{~b}$ & $0,0815 \mathrm{a}$ & $79,3 \mathrm{a}$ & $7,21 \mathrm{a}$ & $93,4 \mathrm{a}$ \\
\hline $6 \times 2 \mathrm{~m}$ & $17,7 \mathrm{a}$ & $13,0 \mathrm{a}$ & $19,3 \mathrm{~b}$ & $0,0882 \mathrm{a}$ & $75,6 \mathrm{a}$ & $6,87 \mathrm{a}$ & $94,4 \mathrm{a}$ \\
\hline $\mathrm{CV}(\%)$ & 6,56 & 6,79 & 8,05 & 20,67 & 12,36 & 12,38 & 4,96 \\
\hline \multirow[b]{2}{*}{ Blocos } & \multicolumn{7}{|c|}{ Variáveis } \\
\hline & $\begin{array}{l}\text { DAP } \\
(\mathrm{cm})\end{array}$ & $\begin{array}{c}\mathrm{H} \\
(\mathrm{m})\end{array}$ & $\begin{array}{c}\mathrm{G} \\
\left(\mathbf{m}^{2} \cdot \mathbf{h} \mathbf{a}^{-1}\right)\end{array}$ & $\begin{array}{c}\mathbf{v} \\
\left(\mathbf{m}^{3}\right)\end{array}$ & $\begin{array}{c}\mathrm{V} \\
\left(\mathrm{m}^{3} \cdot \mathbf{h} \mathbf{a}^{-1}\right)\end{array}$ & $\begin{array}{c}\text { IMA } \\
\left(\mathbf{m}^{3} \cdot \mathbf{h} \mathbf{a}^{-1}\right)\end{array}$ & $\begin{array}{c}\text { S } \\
(\%)\end{array}$ \\
\hline 1 & $15,3 \mathrm{a}$ & $12,6 \mathrm{a}$ & $20,0 \mathrm{~b}$ & 0,0599 a & $75,4750 \mathrm{~b}$ & $0,84 \mathrm{a}$ & $91,4 \mathrm{a}$ \\
\hline 2 & $15,7 \mathrm{a}$ & $13,0 \mathrm{a}$ & $20,9 \mathrm{~b}$ & $0,0707 \mathrm{a}$ & $75,3500 \mathrm{~b}$ & $0,84 \mathrm{a}$ & $95,1 \mathrm{a}$ \\
\hline 3 & $17,6 \mathrm{a}$ & $12,9 \mathrm{a}$ & $25,9 \mathrm{a}$ & $0,0792 \mathrm{a}$ & $96,0250 \mathrm{a}$ & $0,93 \mathrm{a}$ & $93,3 \mathrm{a}$ \\
\hline
\end{tabular}

Médias seguidas de mesma letra na coluna não diferem estatisticamente entre si pelo teste de Tukey a $5 \%$ de probabilidade; $\mathrm{CV}=$ coeficiente de variação. 
competição por luz pode reduzir a sobrevivência, já que é uma espécie heliófita.

O teste de Tukey para agrupamento e separação de médias entre os blocos mostra que houve efeito significativo para área basal $(\mathrm{G})$ e volume total por hectare $(\mathrm{V})$, indicado que o povoamento foi influenciado por fatores edáficos locais. O bloco 3, localizado no lado oeste do povoamento, diferiu dos demais, apresentando as maiores médias de G e V, já os blocos 2 (central) e 3 (leste) produziram efeitos iguais. Essa diferença entre blocos também foi encontrada por Pauletto (2003) na mesma área deste estudo. $\mathrm{O}$ autor estudou a variação espacial do crescimento da teca aos 22 meses em função dos fatores do solo e verificou que o bloco 3 apresentou as maiores concentrações de cálcio, magnésio e matéria orgânica da área experimental, o que proporcionou melhor desenvolvimento da cultura, corroborando os resultados encontrados no presente estudo.

\subsection{Avaliação dos tratamentos aos 16 anos}

Aos 16 anos após o plantio foram observadas diferenças estatísticas, pelo teste de Tukey a 5\% de probabilidade, entre as variáveis DAP, G, v e V (Tabela 3). No entanto, as variáveis $\mathrm{H}$, IMA e $\mathrm{S}$ apresentaram efeitos iguais entre os tratamentos. Essa idade de avaliação permitiu confirmar a tendência de os espaçamentos menores entre plantas acarretam em valores de diâmetro menores, como relatado por Rondon (2006) para T. grandis no médio norte de Mato Grosso e Magalhães et al. (2007) para Eucalyptus no norte de Minas Gerais. Sendo assim, os menores diâmetros resultaram também em menores valores de volume individual nos espaçamentos mais adensados.

Como relatado anteriormente (na idade de 11 anos), o controle local exercido pelos diferentes blocos apresentou efeitos significativos para todas as variáveis avaliadas, com exceção de $\mathrm{S}$. As maiores médias foram observadas nos blocos 3 e 2 , devido às maiores concentrações dos nutrientes cálcio, magnésio e matéria orgânica (Pauletto, 2003).

Foi observado um aumento gradativo dos valores de DAP médio de acordo com o aumento do espaçamento. Porém esses valores foram inferiores aos encontrados por Favalessa et al. (2012) em um povoamento de Tectona grandis de mesma idade submetido a dois desbastes no município de Rosário Oeste, MT, que apresentaram valor médio de 33,65 cm. Rossi et al. (2011), avaliando povoamentos não desbastados de Tectona grandis aos 16 anos em Monte Dourado, Pará, no espaçamento $6 \times 2 \mathrm{~m}$, também obtiveram valores de DAP superior ao deste estudo $(25,3 \mathrm{~cm})$.

Tabela 3. Valores médios de diâmetro a 1,3 m de altura (DAP), altura (H), área basal (G), volume individual (v), volume total por hectare (V), incremento médio anual (IMA) e sobrevivência (S) de Tectona grandis aos 16 anos em Cáceres, MT.

Table 3. Mean values of diameter at $1.3 \mathrm{~m}$ height $(\mathrm{DBH})$, height $(\mathrm{H})$, basal area $(\mathrm{G})$, individual volume (v), total volume per hectare (V), mean annual increment (MAI) and survival (S) Tectona grandis at 16 years old in Cáceres-MT.

\begin{tabular}{|c|c|c|c|c|c|c|c|}
\hline \multirow[b]{2}{*}{ Tratamentos } & \multicolumn{7}{|c|}{ Variáveis } \\
\hline & $\begin{array}{l}\text { DAP } \\
(\mathrm{cm})\end{array}$ & $\begin{array}{c}\mathbf{H} \\
(\mathrm{m})\end{array}$ & $\begin{array}{c}\mathrm{G} \\
\left(\mathbf{m}^{2} \cdot \mathbf{h} \mathbf{a}^{-1}\right)\end{array}$ & $\begin{array}{c}\mathbf{v} \\
\left(\mathbf{m}^{3}\right)\end{array}$ & $\begin{array}{c}\mathrm{V} \\
\left(\mathrm{m}^{3} \cdot \mathbf{h} \mathbf{a}^{-1}\right)\end{array}$ & $\begin{array}{c}\text { IMA } \\
\left(\mathrm{m}^{3} \cdot \mathrm{ha}^{-1}\right)\end{array}$ & $\begin{array}{c} \\
(\%)\end{array}$ \\
\hline $3 \times 2 \mathrm{~m}$ & $15,8 \mathrm{~b}$ & $15,2 \mathrm{a}$ & $27,6 \mathrm{a}$ & $0,0919 b$ & $117,9 \mathrm{a}$ & $7,73 \mathrm{a}$ & $91,0 \mathrm{a}$ \\
\hline $4 \times 2 m$ & $17,5 \mathrm{ab}$ & $14,9 \mathrm{a}$ & $25,5 \mathrm{ab}$ & $0,1081 \mathrm{ab}$ & $101,9 \mathrm{ab}$ & $6,37 \mathrm{a}$ & $92,6 \mathrm{a}$ \\
\hline $5 \times 2 \mathrm{~m}$ & $17,8 \mathrm{ab}$ & $15,1 \mathrm{a}$ & $21,4 \mathrm{~b}$ & $0,1181 \mathrm{ab}$ & $94,8 \mathrm{~b}$ & $5,92 \mathrm{a}$ & $92,3 \mathrm{a}$ \\
\hline $6 \times 2 \mathrm{~m}$ & $19,9 \mathrm{a}$ & $15,8 \mathrm{a}$ & $21,4 \mathrm{~b}$ & $0,1470 \mathrm{a}$ & $93,4 \mathrm{~b}$ & $5,84 \mathrm{a}$ & $92,0 \mathrm{a}$ \\
\hline $\mathrm{CV}(\%)$ & 5,11 & 2,56 & 8,01 & 11,92 & 8,81 & 8,87 & 4,93 \\
\hline \multirow[b]{2}{*}{ Blocos } & \multicolumn{7}{|c|}{ Variáveis } \\
\hline & $\begin{array}{l}\text { DAP } \\
(\mathrm{cm})\end{array}$ & $\begin{array}{c}\mathrm{H} \\
(\mathrm{m})\end{array}$ & $\begin{array}{c}\mathbf{G} \\
\left(\mathbf{m}^{2} \cdot \mathbf{h} \mathbf{a}^{-1}\right)\end{array}$ & $\begin{array}{c}\mathbf{v} \\
\left(\mathbf{m}^{3}\right)\end{array}$ & $\begin{array}{c}\mathrm{V} \\
\left(\mathbf{m}^{3} \cdot \mathbf{h} \mathbf{a}^{-1}\right)\end{array}$ & $\begin{array}{c}\text { IMA } \\
\left(\mathbf{m}^{3} \cdot \mathbf{h} \mathbf{a}^{-1}\right)\end{array}$ & $\begin{array}{c}S \\
(\%)\end{array}$ \\
\hline 1 & $16,6 \mathrm{~b}$ & $14,7 \mathrm{~b}$ & $21,9 \mathrm{~b}$ & $0,0988 \mathrm{~b}$ & $91,8 \mathrm{~b}$ & $5,73 \mathrm{~b}$ & $89,0 \mathrm{a}$ \\
\hline 2 & $17,4 \mathrm{ab}$ & $15,1 \mathrm{~b}$ & $22,5 \mathrm{~b}$ & $0,1101 \mathrm{ab}$ & $91,9 \mathrm{~b}$ & $5,74 \mathrm{~b}$ & $94,3 \mathrm{a}$ \\
\hline 3 & $19,3 \mathrm{a}$ & $15,9 \mathrm{a}$ & $27,5 \mathrm{a}$ & $0,1399 \mathrm{a}$ & $122,4 \mathrm{a}$ & $7,65 \mathrm{a}$ & $92,5 \mathrm{a}$ \\
\hline
\end{tabular}

Médias seguidas de mesma letra na coluna não diferem estatisticamente entre si pelo teste de Tukey a $5 \%$ de probabilidade; $\mathrm{CV}=$ coeficiente de variação. 
As alturas médias foram semelhantes às encontradas por Rossi et al. (2011), em Monte Dourado, PA (14,6 m), e Vendruscolo et al. (2015), em Cáceres, MT, que encontraram valores médios variando de 14 a $15 \mathrm{~m}$. Foram inferiores aos obtidos por Favalessa et al. (2012), em Rosário Oeste, MT (18,9 m), e Chaves (2013), em Indiavaí, MT (20,6 m). Constatou-se estatisticamente igualdade para as médias das alturas entre os diferentes tratamentos nas diferentes idades, resultado que corrobora com os obtidos por Berger et al. (2002), Rondon (2006) e Araújo et al. (2014) avaliando o crescimento em altura das espécies Eucalyptus saligna, Tectona grandis e Eremanthus incanus, respectivamente, a qual não foi influenciada pelo espaçamento.

O comportamento das variáveis área basal $(\mathrm{G})$ e volume total por hectare $(\mathrm{V})$ foi semelhante ao observado por Scolforo (1998) e Sartório (2014), apresentando maiores valores nos espaçamentos mais adensados, evidenciando maior ocupação da área nessas condições. Os valores médios de $\mathrm{G}$ e $\mathrm{V}$ foram inferiores aos obtidos por Rossi et al. (2011) para povoamentos de teca de mesma idade em Monte Dourado, PA, com $\mathrm{G}=48,2 \mathrm{~m}^{2}$ e $\mathrm{V}=310,2 \mathrm{~m}^{3}$.

O volume individual (v) das árvores aos 16 anos também apresentou médias inferiores se comparadas aos valores do estudo de Rossi el al. (2011) $\left(\mathrm{v}=0,3720 \mathrm{~m}^{3}\right)$. Consequentemente, o incremento médio anual (IMA) apresentou-se baixo, denotando que, devido à não ocorrência de desbastes, o incremento e a produção dos povoamentos foram afetados negativamente.

Em relação ao percentual de sobrevivência (S) da teca nos diferentes espaçamentos, os resultados apresentaram-se acima de 90\%, com leve decréscimo em relação ao observado na idade anterior de avaliação (11 anos). A sobrevivência ( $\mathrm{S} \%$ ) foi superior à observada por Macedo et al. (2005) no noroeste do estado de Minas Gerais, com S\% igual a 70,4\%, Ribeiro et al. (2006) em Minas Gerais, em diferentes níveis de adubação e com S\% entre 63,8\% e 90,0\%, e inferior ao observado por Pelissari et al. (2013) na região de Nossa Senhora do Livramento, MT, com S\% de 96,8\%. Macedo et al. (2005) citam que a porcentagem de sobrevivência das espécies florestais fornece informações sobre o potencial de adaptação em um determinado local. Desse modo, a sobrevivência observada neste estudo demonstra uma boa adaptabilidade da teca às condições edafoclimáticas da região de Cáceres, MT.

Os baixos valores médios das variáveis dendrométricas avaliadas neste estudo estão relacionados à ausência de desbastes até a idade em questão. Estudos desenvolvidos por Cruz et al. (2008) na região de Tangará da Serra, MT, revelou que o primeiro desbaste em povoamento de teca deve ser feito antes de 6 anos, para evitar o início da competição e assim garantir o ritmo de crescimento da cultura. Segundo Pelissari et al. (2013), a competição entre as árvores em espaçamento reduzido acarreta em primeira ordem a redução do crescimento em diâmetro, enquanto o crescimento em altura é pouco afetado. Garcia (2006) e Pelissari et al. (2013) destacam que a variável DAP responde proporcionalmente à intensidade do desbaste aplicado em povoamentos de teca, proporcionando assim maiores taxas de incremento e de produção.

Dentre os espaçamentos avaliados, verificou-se que a densidade de 1.250 árv.ha-1 ${ }^{-1}$ que corresponde ao espaçamento $4 \times 2 \mathrm{~m}$, apresentou igualdade estatística entre os tratamentos com as maiores médias de diâmetro e produção volumétrica de madeira, obtendo-se nele o melhor desempenho nas condições avaliadas.

\section{CONCLUSÕES}

Aos 11 anos de idade os espaçamentos testados resultaram em efeitos significativos apenas para a área basal, que apresentou as maiores médias nos espaçamentos mais densos ( $3 \times 2 \mathrm{~m} \mathrm{e} 4$ × $2 \mathrm{~m}$ ).

Aos 11 e 16 aos anos observou-se que quanto menor a densidade de árvores, maiores foram os valores das variáveis DAP e volume individual, e que quanto maior a densidade de árvores, maiores foram os valores observados para as variáveis área basal e volume total por hectare.

Dentre os espaçamentos avaliados recomenda-se a densidade 1.250 árv.ha $^{-1}$, que corresponde ao espaçamento $4 \times 2 \mathrm{~m}$, por apresentar igualdade estatística entre as maiores médias de diâmetro e produção volumétrica total semelhante ao espaçamento com maior estoque de madeira $(3 \times 2 \mathrm{~m})$.

Os povoamentos avaliados apresentaram médias dendrométricas e produção inferior quando comparados aos povoamentos comerciais de teca no estado devido à não ocorrência de desbastes. 
STATUS DA SUBMISSÃO

Recebido: 17 abr., 2015

Aceito: 16 fev., 2016

\section{AUTOR(ES) PARA CORRESPONDÊNCIA}

\section{Diogo Guido Streck Vendruscolo}

Departamento de Engenharia Florestal, Universidade Federal de Mato Grosso - UFMT, Av. Fernando Corrêa da Costa, 2367, Boa Esperança, CEP 78060-900, Cuiabá, MT, Brasil e-mail: diogoguido@hotmail.com

\section{REFERÊNCIAS}

Araújo JG, David HC, Morais VA, Scolforo JRS, Netto SP, Zanette VH et al. Crescimento em diâmetro e altura da regeneração natural de Eremanthus incanus. Floresta 2014; 44(2): 217-228. http://dx.doi.org/10.5380/rf.v44i2.31362.

Associação Brasileira de Produtores de Florestas Plantadas - ABRAF. Anuário Estatístico da ABRAF 2013 - ano base 2012. Brasília; ABRAF; 2013.

Bartoszeck ACPS, Machado AS, Figueiredo A Fo, Oliveira EB. Dinâmica da relação hipsométrica em função da idade, do sítio e da densidade inicial de povoamentos de bracatinga da região metropolitana de Curitiba, Paraná. Revista Árvore 2004; 28(4): 517-533. http://dx.doi. org/10.1590/S0100-67622004000400006.

Berger R, Schneider PR, Finger CAG, Haselein CR. Efeito do espaçamento e da adubação no crescimento de um clone de Eucalyptus saligna Smith. Ciência Florestal 2002; 12(2): 75-87.

Camino R, Morales JP, editores. Las plantaciones de teca em América Latina: mitos y realidades. Turrialba: CATIE; 2013. 392 p. Informe Técnico n. 397.

Chaves AGS. Modelagem do crescimento e da produção de Tectona grandis L.f. até a idade de rotação. [dissertação]. Cuiabá: Departamento de Engenharia Florestal, Universidade Federal de Mato Grosso; 2013.

Cruz JP, Leite HG, Soares CB, Campos JCC, Smit L, Nogueira GS. Curvas de crescimento e de índice de local para povoamentos de Tectona grandis em Tangará da Serra, Mato Grosso. Revista Árvore 2008; 32(4): 679-685. http://dx.doi.org/10.1590/S0100-67622008000400009.

Curtis RO. Height diamenter and height diamenter age equations for second growth Douglas-fir. Forest Science 1967; 13(4): 356-375.

Favalessa CMC, Ubialli JA, Caldeira SF, Drescher R, Acosta FC. Equações de sortimentos para Tectona grandis na região centro - sul de Mato Grosso. Pesquisa
Florestal Brasileira 2012; 32(72): 389-399. http://dx.doi. org/10.4336/2012.pfb.32.72.389.

Figueiredo EO. Avaliação do crescimento da teca (Tectona grandis. Lf.) para análise de tronco. Rio Branco: Embrapa Acre; 2001. p. 4. Embrapa Acre Instruções Técnicas n. 35).

Garcia ML. Intensidade de desbastes em um povoamento de Tectona grandis L.f., no município de Sinop-MT. [dissertação]. Cuiabá: Departamento de Engenharia Florestal, Universidade Federal de Mato Grosso; 2006.

Krishnapillay B. Silvicultura y ordenación de plantaciones de teca. Unasylva 2000; 51: 14-21.

Macedo RLG, Gomes JE, Venturin N, Salgado BG. Desenvolvimento inicial de Tectona grandis L.f. (teca) em diferentes espaçamentos no município de Paracatu, MG. Cerne 2005; 11(1): 61-69.

Magalhães WM, Macedo RLG, Venturin N, Higashikawa EM, Yoshitani M Jr. Desempenho silvicultural de clones e espécies/procedências de Eucalyptus na região Noroeste de Minas Gerais. Cerne 2007; 13(4): 368-375.

Neves SMAS, Nunes MCM, Neves RJ. Caracterização das condições climáticas de Cáceres/MT Brasil, no período de 1971 a 2009: subsídio às atividades Agropecuárias e turísticas municipais. Boletim Goiano de Geografia 2011; 31(2): 55-68.

Oliveira FGR, Sousa GTO, Azevedo GB, Barreto PAB. Desempenho de modelos hipsométricos para um povoamento de Eucalyptus urophylla no município de Jaguaquara, Bahia. Enciclopédia Biosfera 2011; 7(13): 331-338.

Passos CAM, Bufulin L, Gonçalves MR. Avaliação silvicultural de Tectona grandis L.f em Cáceres - MT. Ciência Florestal 2006; 16(2): 225-232.

Pauletto D. Variações espaciais do crescimento e de solo para um plantio de Tectona grandis L. $f$ no município de Cáceres - MT [monografia]. Cuiabá: Departamento de Engenharia Florestal, Universidade Federal de Mato Grosso; 2003.

Pelissari AL, Caldeira SF, Drescher R. Desenvolvimento quantitativo e qualitativo de Tectona grandis L.f. em Mato Grosso. Floresta e Ambiente 2013; 20(3): 371-383. http:// dx.doi.org/10.4322/floram.2013.027.

Ribeiro A, Ferraz AC Fo, Mello JM, Ferreira MZ, Lisboa PMM, Scolforo JRS. Estratégias e metodologias de ajuste de modelos hipsométricos em plantios de Eucalyptus sp. Cerne 2010; 16(1): 22-31. http://dx.doi.org/10.1590/ S0104-77602010000100003.

Ribeiro FA, Macedo RLG, Venturim N, Morais VM, Gomes JE, Yoshitami M Jr. Feitos da adubação de plantio sobre o estabelecimento de mudas de Tectona grandis L.f. (teca). Revista Científica Eletrônica de Engenharia Florestal 2006; 4(7): 1-13.

Rondon EV. Estudo de biomassa de Tectona grandis L.f. sob diferentes espaçamentos no estado de Mato Grosso. 
Revista Árvore 2006; 30(3): 337-341. http://dx.doi. org/10.1590/S0100-67622006000300003.

Rossi AS, Drescher R, Pelissari AL, Lanssanova LR. Relação hipsométrica e crescimento de Tectona grandis L.f. no município de Monte Dourado PA. Scientia Forestalis 2011; 39(91): 301-307.

Sartório IP. Avaliação e modelagem do crescimento de florestas energéticas de eucalipto plantadas em diferentes densidades [dissertação]. Curitiba: Departamento de Engenharia Florestal, Universidade Federal do Paraná; 2014.

Schuhli GS, Paludzyszyn EO Fo. Cenário da silvicultura de teca e perspectivas para o melhoramento genético. Pesquisa Florestal Brasileira 2010; 30(63): 217-230. http:// dx.doi.org/10.4336/2010.pfb.30.63.217.

Scolforo JRS. Modelagem do crescimento e da produção de florestas plantadas e nativas. Lavras: UFLA/FAEPE; 1998.

Shimizu JY, Klein H, Oliveira JRV. Diagnóstico das plantações florestais em Mato Grosso. Cuiabá: Central de Texto; 2007.
Silva FAZ, Azevedo CAV. Versão do programa computacional Assistat para o sistema operacional Windows. Revista Brasileira de Produtos Agroindustriais 2002; 4(1): 71-78. http://dx.doi.org/10.15871/1517-8595/rbpa.v4n1p71-78.

Souza JT. Influência de diferentes espaçamentos no valor total do estoque madeireiro de um povoamento de teca (Tectona grandis L. f.) com 11 anos de idade, Cáceres$M T$ [dissertação]. Seropédica: Instituto de Florestas, Universidade Federal Rural do Rio de Janeiro; 2010.

Vendruscolo DGS, Chaves AGS, Silva RS, Souza HS, Medeiros RA, Motta AS et al. Identidade em modelos hipsométricos para Tectona grandis com diferentes espaçamentos em Cáceres-MT. Nativa 2015; 03(1): 4348. http://dx.doi.org/10.14583/2318-7670.v03n01a07.

Vendruscolo DGS, Silva RS, Souza HS, Chaves AGS, Motta AS. Modelos volumétricos para teca em diferentes espaçamentos em Cáceres, Mato Grosso. Enciclopédia Biosfera 2014; 10(19): 1333-1341. 